\title{
INCOMPRESSIBLE FLOW IN GRANULATED POROUS MEDIA WITH NULL INITIAL VELOCITY
}

José Luiz Boldrini

Departamento de Matemática Aplicada - IMECC

UNICAMP, Campinas, SP

João Paulo Lukaszczyk

Departamento de Matemática - CCNE

UFSM - Santa Maria, RS.

\section{RESUMO}

Neste trabalho nós estudamos uma equação do tipo NavierStokes que modela o fluxo de um fluido viscoso, homogêneo e incompressível num meio poroso granular (não consolidado) .Usando argumentos do tipo ponto fixo obtemos condições para a existência de solução em espaços de Hölder.

\section{ABSTRACT}

In this work we study a Navier-Stokes type equation which models the flow of a viscous, homogeneous and incompressible fluid in a isotropic granular (non consolidated) porous media. Using point fixed type arguments we obtain conditions for existence of solution for the equation in Hölder's spaces. 


\section{INTRODUCTION}

In this work we will show results of existence of solution for the following system which represents the flow of a fluid in a granulated porus media, for example, if we put water in a box of sand, the water will flow through the sand with the action of gravity.

$$
\left\{\begin{array}{l}
\rho u_{t}+\rho \cdot \nabla\left(\frac{u}{n}\right)-\mu \Delta u+n \nabla p+\mu F(n) u=\rho n g, \text { in } Q_{T} \\
\operatorname{div} u=0, \text { in } Q_{T} \\
u(x, 0)=0, \forall x \in \Omega \\
u(x, t)=0, \forall t \in(0, T) \forall x \in \partial \Omega
\end{array}\right.
$$

Here $Q_{T}=\Omega \times(0, T), \Omega \subset I R^{d} ; \mathrm{d}=2,3$ is a bounded domain. $u(x, t)$ denotes the velocity of the fluid. $p(x, t)$ is hydrostatic pressure at point $x$ and instant $t$. For reasons of uniqueness in this paper we will suppose that in each instant of time the pressure has average zero, that is, $\int_{\Omega} p(x, t) d x=0, \forall t \in[0, T] . \mu$ is viscosity of fluid. $\rho$ is density of fluid, here, without lost of generality in the case of homogeneous fluids, we have supposed constant equal to one. $n(x, t)$ is the porosity of granular porus media, that is, the volume of empty space divided by the total volume of a portion of $\Omega$ in the neighborhood of $x$. The variation of porosity varies from zero to one. When the porosity becomes null then porous media is solid and it can be excluded from the drain region, and when porosity becomes one in a certain sub region this mean that there is no granular material, and the flow is free. In this way we can always suppose $0<n(x, t) \leq 1$. F represents the frictional effects introduced by the presence of the porous environment. We will suppose that $F$ satisfies $\lim _{z \rightarrow 1} F(z)=0$ and $\lim _{z \rightarrow 0} F(z)=\infty$ which is consistent wiht the meaning of $F . g$ is an external force field. 


$$
\Delta u=\left(\Delta u_{1}, \ldots, \Delta u_{d}\right) \text { and }(u \cdot \nabla u)_{i}=\sum_{j=1}^{d} u_{j} \frac{\partial u_{i}}{\partial x_{j}}, i=1,2, \ldots, d
$$

Essentially, the system above comes from the classical NavierStokes equations, considering the new elements in the problem (porosity and friction) and making average integrals in small volumes. Then, the variables $u$ and $p$ are macroscopic averages of real velocity and pressure of fluid particles. For details see [4].

Our main result was obtained supposing that the porosity is a function of the pressure, which is more realistic and essentially is the following result:

If $g=g_{1}+\nabla \Psi, n(x, t)=n_{o}+\delta f(p(x, t))$ where $0<n_{o}<1, \delta$ is a positive constant taken small (in a certain sense), $g_{1} \in \mathrm{H}^{\lambda}\left(Q_{T}\right)$, $\Psi \in \mathrm{H}^{1+\lambda}\left(Q_{T}\right)$ and $f$ is a adequate function then exist solution $(u, p)$ of (1) in which $p \in \mathrm{H}^{1+\lambda}\left(Q_{T}\right) ; u \in\left(\mathrm{H}^{2+\lambda}\left(Q_{T}\right)\right)^{d}$. Here $\mathrm{H}^{k+\lambda}\left(Q_{T}\right)$, $k \in I N \quad$ is a Hölder's space of $k+\lambda$ order (see preliminaries in section 2). With $p$ and $u$ satisfying $\|p-\Psi\|_{1+\lambda}<R$ and $\|u\|_{2+\lambda}<R$ where $\delta>0, \quad R>0$ and $\left\|g_{1}\right\|_{\lambda}$ should be sufficiently small and $\mu C_{s} F\left(n_{o}\right)<n_{o}$. Here $C_{s}$ is the constant of Solonnikov's Theorem of existence and uniqueness (see preliminaries bellow).

The existence of solutions will be gotten by means of an adaptation of Schauder's fixed point Theorem.

The solution was searched at Hölder's spaces because, until now, the estimates in Sobolev's spaces are not sufficient to control the nonlinear terms in the pressure, more precisely, for the velocity we get adequate compact immersions but for the pressure we do not. 
Like in classical Navier-Stokes equations it's possible to obtain a result of uniqueness, but with more restriction at the smallness of the data above.

The work is organized in the following way:

In section 2 we recall Hölder's spaces and write down some basic results (the principal of them is an existence and uniqueness Solonnikov's theorem). We state our two basic theorems and prove one lemma which will be used in section 3.and in section 3 we will prove the existence theorem. Like is usual in PDE problems, we will denote by $C$ a generic, positive constant depending only on $\Omega$ and the data of the problem.

\section{PRELIMINARIES AND RESULTS}

Let $\Omega \subset I R^{d}$ be an open and $\lambda \in I R \quad, 0<\lambda \leq 1, m \in\{0,1,2, \ldots\}$.

We define:

$\mathrm{H}^{m+\lambda}(\Omega)=\left\{u: \Omega \rightarrow I R\right.$ which are $C^{m}(\bar{\Omega})$ with $\left.\|\mathrm{u}\|_{\mathrm{H}^{m+\lambda}(\Omega)}<\infty\right\}$ where

$$
\|\mathrm{u}\|_{H^{m+\lambda}(\Omega)}=\sum_{\alpha \leq m} \sup _{\Omega}\left|D^{\alpha} u(x)\right|+\sum_{\alpha=m}\left(\sup _{x, y \in \Omega} \frac{\left|D^{\alpha} u(x)-D^{\alpha} u(y)\right|}{|x-y|^{\lambda}}\right)
$$

The spaces above are well known and one reference about this is [1].

The spaces below are, somewhat, generalizations of ordinary Hölder's spaces:

Let $\lambda \in I R, 0<\lambda \leq 1, m \in\{0,1,2, \ldots\}$. and following the notation used in [4] we define: 
$\mathrm{H}^{2 m+\lambda}\left(Q_{T}\right)=\left\{\begin{array}{c}u: \bar{\Omega} \times[0, T] \rightarrow I R \text { where } u(\cdot, t) \in \mathrm{C}^{2 \mathrm{~m}}(\bar{\Omega}) \forall \mathrm{t} \in[0, \mathrm{~T}] \text { and } \\ u(x, \cdot) \in \mathrm{C}^{\mathrm{m}}[0, \mathrm{~T}] \forall \mathrm{x} \in \bar{\Omega} \text { with }\|\mathrm{u}\|_{\mathrm{H}^{2 m+\lambda}\left(Q^{T}\right)}<\infty\end{array}\right\}$

where

$$
\begin{aligned}
& \|\mathrm{u}\|_{\mathrm{H}^{2 m+\lambda}\left(Q_{T}\right)}=\sum_{\alpha \leq 2 m} \sup _{\Omega}\left|D_{x}^{\alpha} u(x, t)\right|+\sum_{k=1}^{m} \sup _{Q_{T}}\left|D_{t}^{k} u(x, t)\right|+ \\
& +\sum_{\alpha=2 m}\left(\sup _{x, y, t} \frac{\left|D_{x}^{\alpha} u(x, t)-D_{x}^{\alpha} u(y, t)\right|}{|x-y|^{\lambda}}+\sup _{x, t . s} \frac{\left|D_{x}^{\alpha} u(x, t)-D_{x}^{\alpha} u(x, s)\right|}{|t-s|^{\frac{\lambda}{2}}}\right)+ \\
& +\sup _{x, y, t} \frac{\left|D_{t}^{m} u(x, t)-D_{t}^{m} u(y, t)\right|}{|x-y|^{\lambda}}+\sup _{x, t . s} \frac{\left|D_{t}^{m} u(x, t)-D_{t}^{m} u(x, s)\right|}{|t-s|^{\frac{\lambda}{2}}} \\
& \mathrm{H}^{1+\lambda}\left(Q_{T}\right)=\left\{\begin{array}{c}
u: \bar{\Omega} \times[0, T] \rightarrow I R \text { where } u(\cdot, t) \in \mathrm{C}^{1}(\bar{\Omega}) \forall \mathrm{t} \in[0, \mathrm{~T}] \text { and } \\
u(x, \cdot) \in \mathrm{C}^{0}[0, \mathrm{~T}] \forall \mathrm{x} \in \bar{\Omega} \text { with }\|\mathrm{u}\|_{\mathrm{H}^{1+\lambda}\left(Q^{T}\right)}<\infty
\end{array}\right\}
\end{aligned}
$$

where

$$
\begin{aligned}
& \|\mathrm{u}\|_{H^{1+\lambda}\left(Q_{i}\right)}=\sum_{\alpha \leq 1} \sup _{\Omega}\left|D_{x}^{\alpha} u(x, t)\right|+\sum_{\alpha=1} \sup _{x, y, t} \frac{\left|D_{x}^{\alpha} u(x, t)-D_{x}^{\alpha} u(y, t)\right|}{|x-y|^{\lambda}} \\
& +\sum_{\alpha=1} \sup _{\text {x.t.s }} \frac{\left|D_{x}^{\alpha} u(x, t)-D_{x}^{\alpha} u(x, s)\right|}{|t-s|^{\frac{\lambda}{2}}}
\end{aligned}
$$

For the sake of simplicity we will denote: $\|\mathrm{u}\|_{\mathrm{H}^{m+\lambda}(\Omega)}=\|u\|_{m+\lambda} ;\|\mathrm{u}\|_{\mathrm{H}^{2 m+\lambda}\left(Q_{T}\right)}=\|u\|_{2 m+\lambda}$.

and $\|\mathrm{u}\|_{\mathrm{H}^{1+\lambda}\left(Q_{I}\right)}=\|u\|_{1+\lambda}$ because from context will be clear the use of one or another norm. 
For functions with vectorial values, like $u=\left(u_{1}, \ldots, u_{d}\right)$ we say $u$ is a Hölder's space function of order $m+\lambda$ if $u \in\left(\mathrm{H}^{m+\lambda}\left(Q_{T}\right)\right)^{d}$ and $\|\mathrm{u}\|_{\left(\mathrm{H}^{m+\lambda}\left(Q_{T}\right)\right)^{t}}=\sum_{k=1}^{d}\left\|\mathrm{u}_{\mathrm{k}}\right\|_{m+\lambda}$. This is a similar form like in Navier-Stokes standard theory and we will not make different notations for scalar or vectorial functions for $m+\lambda$ norms.

Another important property of these spaces is:

$$
\|\mathrm{u} \cdot \mathrm{v}\|_{j+\lambda} \leq C(j)\|\mathrm{u}\|_{j+\lambda}\|\mathrm{v}\|_{j+\lambda} ; \forall u, v \in \mathrm{H}^{j+\lambda}(\Omega)\left(\operatorname{or~H}^{j+\lambda}\left(Q_{T}\right)\right)
$$

where, in the case of functions defined over $\Omega$ we have $j \in I N$, and in the case of functions defined over $Q_{T}$ we have $j=1$ or $j=2 m$, with $m \in I N$.

We have the following results of immersion for the Hölder's spaces:

Theorem 1: Let $m$ be a non negative integer and $\Omega$ be a bounded open of $I R^{d}, d \in I N, T>0$ and $0<v<\lambda \leq 1$. Then the following immersions are continuous and compact:

$$
\begin{aligned}
& (i) \mathrm{H}^{m+\lambda}(\Omega) \rightarrow C^{m}(\bar{\Omega}) \\
& (\text { ii }) \mathrm{H}^{m+\lambda}(\Omega) \rightarrow \mathrm{H}^{m+v}(\Omega) \\
& (\text { iii }) \mathrm{H}^{2 m+\lambda}\left(Q_{T}\right) \rightarrow C^{2 m, m}\left(\overline{Q_{T}}\right) \\
& (\text { iv }) \mathrm{H}^{2 m+\lambda}\left(Q_{T}\right) \rightarrow \mathrm{H}^{2 m+\lambda} \\
& (v) \mathrm{H}^{1+\lambda}\left(Q_{T}\right) \rightarrow C^{1,0}\left(\overline{Q_{T}}\right)
\end{aligned}
$$

The demonstrations of (i) and (ii) can be found in [1] and the proofs of (iii) to ( $v$ ) are quite similar making use of Arzela-Ascoli's Theorem. 
The notation $C^{m, n}\left(\overline{Q_{T}}\right)$ means functions which are $C^{m}$ in space variables and $C^{n}$ at time variables. Here $m$ and $n$ are integers not negatives.

Let's recall the following spaces from classical Navier-Stokes theory (see Teman [6] and Solonnikov [5])

$$
\begin{aligned}
& J_{q}(\Omega)=\left\{u \in\left(L^{q}(\Omega)\right)^{d}:\left.u \cdot \vec{N}\right|_{\partial \Omega} \equiv 0 \text { and } \operatorname{div} u=0\right\} \\
& G_{q}(\Omega)=\left\{u \in\left(L^{q}(\Omega)\right)^{d}: u=\nabla \Phi\right\}
\end{aligned}
$$

Observe that $J_{2}(\Omega)=H$ and $G_{2}(\Omega)=H^{\perp}$. We have $L^{q}(\Omega)=J_{q}(\Omega) \oplus G_{q}(\Omega)$ and this define continuous projections $P_{J_{q}}$ and $P_{G_{q}}$ (with norm depending only on $\Omega$ and $q$ ). Indicating $P: L^{2}(\Omega) \rightarrow H \quad$ we have for $f \in\left(L^{2}(\Omega)\right)^{d} \cap\left(L^{q}(\Omega)\right)^{d}$ that $P_{J_{q}}(f)=P(f)$ This induce continuous projection from $L^{p}\left(0, T,\left(L^{q}(\Omega)\right)^{d}\right)$ into $L^{p}\left(0, T, J_{q}(\Omega)\right)$ $P_{J_{q}}(\mathrm{u})(\mathrm{x}, \mathrm{t})=P_{J_{u}}(\mathrm{u}(\cdot, \mathrm{t}))(\mathrm{x})$. In the context of Hölder's spaces we have similar things:

$$
\begin{aligned}
& J^{m+\lambda}(\Omega)=\left\{u \in\left(\mathrm{H}^{m+\lambda}(\Omega)\right)^{d}:\left.u \cdot \vec{N}\right|_{\partial \Omega} \equiv 0 \text { and divu }=0\right\} \\
& G^{m+\lambda}(\Omega)=\left\{u \in\left(\mathrm{H}^{m+\lambda}(\Omega)\right)^{d}: u=\nabla \Phi\right\}
\end{aligned}
$$

$\mathrm{H}^{m+\lambda}(\Omega)=J^{m+\lambda}(\Omega) \oplus G^{m+\lambda}(\Omega)$ which define continuous projections into $J^{m+\lambda}(\Omega)$ and $G^{m+\lambda}(\Omega)$. 
Due the regularity at $\partial \Omega$ and because $\Omega$ is bounded we have $P_{J_{u}}(\mathrm{f})=P(f) \quad$ for $\quad \mathrm{f} \in\left(\mathrm{L}^{2}(\Omega)\right)^{d} \cap\left(\mathrm{H}^{m+\lambda}(\Omega)\right)^{d}$. This induce continuous projection $P$ at $\left(\mathrm{H}^{m+\lambda}\left(Q_{T}\right)\right)^{d}$ defined by $P(\mathrm{u})(\mathrm{x}, \mathrm{t})=(\mathrm{Pu}(\cdot, \mathrm{t}))(\mathrm{x})$. With this definition $P$ commute with $\partial / \partial t$ operator and then $P\left(\left(\mathrm{H}^{m+\lambda}\left(Q_{T}\right)\right)^{d}\right) \subset\left(\mathrm{H}^{m+\lambda}\left(Q_{T}\right)\right)^{d}$ and we have, for each $t, P(\mathrm{u})(\cdot, \mathrm{t}) \in J^{m+\lambda}(\Omega)$ and

$$
\|P(u)\|_{m+\lambda} \leq C\|u\|_{m+\lambda} ; \forall u \in\left(\mathrm{H}^{m+\lambda}\left(Q_{T}\right)\right)^{d}
$$

where $C=C(\Omega, m, \lambda)$. More details can be found [7].

The following theorem due to Solonnikov [5] will be very important for our arguments:

Theorem 2: Let $\Omega \subset I R^{d}$ be a bounded domain with $\partial \Omega \in \mathrm{H}^{2+\lambda}(\Omega)$ (that is $\partial \Omega$ can be locally mapped by a function $\mathrm{H}^{2+\lambda}(\Omega)$ class $), \mathrm{v}_{\mathrm{o}} \in\left(\mathrm{H}^{2+\lambda}(\Omega)\right)^{d}$ and $\mathrm{f} \in\left(\mathrm{H}^{\lambda}\left(Q_{T}\right)\right)^{d}$ satisfying the following conditions: $\mathrm{P}(\mathrm{f}) \in\left(\mathrm{H}^{\lambda}\left(Q_{T}\right)\right)^{d} ; \operatorname{div} v_{o} \equiv 0 ;\left.v_{o}\right|_{\partial \Omega} \equiv 0$ and

$$
P\left(f(x, 0)+\mu \Delta v_{o}(x)\right)=0, \forall x \in \partial \Omega .
$$

Then the problem:

$$
\left\{\begin{array}{l}
\rho v_{t}-\mu \Delta v+\nabla p=f \text { in } Q_{T} \\
\operatorname{div} v=0, \text { in } Q_{T} \\
v(x, 0)=v_{o}(x), \forall x \in \Omega \\
v(x, t)=0, \forall t \in(0, T) \forall x \in \partial \Omega
\end{array}\right.
$$


has a unique solution $(v, p)$ where $\quad \mathrm{v} \in\left(\mathrm{H}^{2+\lambda}\left(Q_{T}\right)\right)^{d}$ and $\nabla \mathrm{p} \in\left(\mathrm{H}^{\lambda}\left(Q_{T}\right)\right)^{d}$ and we have

$\|v\|_{2+\lambda}+\|\nabla p\|_{\lambda} \leq C_{1}\left(\|f\|_{\lambda}+\left\|P_{J}(f)\right\|_{\lambda}+\left\|v_{o}\right\|_{2+\lambda}\right)+$

$C_{2} \max _{Q_{T}}|v(x, t)|$

The constants $C_{1}$ and $C_{2}$ do not depend on $T$

Observation 1: The reason of hypothesis (6) is for a consistency condition.

Observation 2: The result in [5] is somewhat more general than that stated above. The equation considered in [5] is $\rho v_{t}-\mu \Delta v+a(x, t) v+\sum_{k=1}^{3} a_{k}(x, t) \frac{\partial v}{\partial x_{k}}+\nabla p=f(x, t)$ where $\quad a$ and $a_{k}$ are certain given matrices. Here we consider $a \equiv a_{k} \equiv 0$.

Observation 3: Using of Close Graph Theorem we have: $\sup |v(x, t)| \leq C\|f\|_{\lambda}$.

$Q_{I}$

Observation 4: With the hypothesis that pressure has average zero, that is, $\int_{\Omega} p(x, t) d x=0, \forall t \in[0, T]$ then we have (by the mean value Theorem of calculus) that $\sup |p(x, t)| \leq \sup |\nabla p(x, t)|$ in this way $p \in \mathrm{H}^{1+\lambda}\left(Q_{T}\right)$. $Q_{T}$ $Q_{T}$

From of this observations, and by (5) we can rewrite (7) in the following form:

$$
\|v\|_{2+\lambda}+\|p\|_{1+\lambda} \leq C\left(\|f\|_{\lambda}+\left\|v_{o}\right\|_{2+\lambda}\right)
$$

Here $C$ does not depend on $T$.

The following theorem is the basic result obtained in the theory. 
Theorem 3 (existence of solution): Let $\Omega \subset I R^{d}$ be a bounded domain with $\partial \Omega \in \mathrm{H}^{2+\lambda}, \quad \mathrm{v}_{\mathrm{o}} \in\left(\mathrm{H}^{2+\lambda}(\Omega)\right)^{d}$. Let $f: I R \rightarrow I R$ be a local Lipschitz function with derivative also local Lipschitz satisfying $f(0)=0$. Let $F:(0,1) \rightarrow I R$ a local Lipschitz function satisfying $\lim _{z \rightarrow 1} F(z)=0$ and $\lim _{z \rightarrow 0} F(z)=\infty$. Let $g=g_{1}+g_{2}$ be a function where $g_{1} \in \mathrm{H}^{\lambda}\left(Q_{T}\right)$ satisfying $g_{1}(x, 0)=0, \forall x \in \Omega$ and $g_{2}=\nabla \Psi$ with $\Psi \in \mathrm{H}^{1+\lambda}\left(Q_{T}\right)$ and $\int_{\Omega} \Psi(x, 0) d x=0$. Then for $\delta>0, R>0$ and $\left\|g_{1}\right\|_{\lambda}$ sufficiently small with $\mu C_{s} F\left(n_{0}\right)<n_{0}$, the problem (1) with $n=n_{0}+\delta f(p)$ has a solution $(u, p) \in\left(\mathrm{H}^{2+\lambda}\left(Q_{T}\right)\right)^{d} \times \mathrm{H}^{1+\lambda}\left(Q_{T}\right) \quad$ with $\|u\|_{2+\lambda}<R$, $\|p-\Psi\|_{1+\lambda}<R$ and $p(x, 0)=\Psi(x, 0)$.

Observation 1: Only for physical reasons we consider the dimension to be 2 or 3. The argument is still valid in higher dimensions.

Observation 2: The condition $\mu C_{s} F\left(n_{0}\right)<n_{0}$ tell us that exist a basic porosity $\bar{n}$ for which $\forall n_{0}>\bar{n}$ we have $\mu C_{s} F\left(n_{0}\right)<n_{0}$. A possible interpretation for this is: We guarantee the existence of solution when the porosity is close to one (when the equation is close to pure Navier-Stokes equation, $n=1$ ).

Observation 3: We define a function $f: \Omega \rightarrow I R$ to be local Lipschitz in the sense that for every open set $\omega \subset \Omega$ with $\bar{\omega}$ strictly contained in $\Omega$ we have $\left.f\right|_{\omega}$ a Lipschitz function. 
The following lemma will be useful to demonstrate the above theorem, more precisely, to get uniform limitations for the aproximations of $u$ and $p$.

Lemma 1: Given $\delta>0, R>0,0<n_{0}<1, f: I R \rightarrow I R$ a local Lipschitz function with derivative also local Lipschitz satisfying $f(0)=0$ and $F:(0,1) \rightarrow I R$ a local Lipschitz function satisfying $\lim _{z \rightarrow 1} F(z)=0$ and $\lim _{z \rightarrow 0} F(z)=\infty$ and $g \in \mathrm{H}^{\lambda}\left(Q_{T}\right)$. Then for $\|u\|_{1+\lambda}<R$ and $\|p-\Psi\|_{1+\lambda}<R$ we have:

i) $\|f(p)\|_{\lambda}<C\|p\|_{\lambda}$

ii) $\left\|f^{\prime}(p)\right\|_{\lambda}<\left|f^{\prime}(0)\right|+C\left(R+\|\Psi\|_{1+\lambda}\right)$

iii) $\left\|F\left(n_{0}+\delta f(p)\right)\right\|_{\lambda}<F\left(n_{0}\right)+\delta C\left(R+\|\Psi\|_{1+\lambda}\right)$

iv) $n_{o}+\delta f(p(x, t)) \geq \frac{n_{o}}{2} ; \forall(x, t) \in Q_{T}$ if,

and only if, $\delta \leq \frac{n_{0}}{2 C\left(R+\|\Psi\|_{1+\lambda}\right)}$

v) If $n_{o}+\delta f(p) \geq \frac{n_{o}}{2}$ then $\left\|\frac{1}{n_{o}+\delta f(p)}\right\|_{\lambda} \leq C+\delta C\left(R+\|\Psi\|_{1+\lambda}\right)$

$v i)\left\|\left(n_{o}+\delta f(p)\right) g\right\|_{\lambda} \leq n_{o}\|g\|_{\lambda}+\delta C\left(R+\|\Psi\|_{1+\lambda}\right)\|g\|_{\lambda}$

vii $)\|f(p) \nabla(p-\Psi)\|_{\lambda} \leq R C\left(R+\|\Psi\|_{1+\lambda}\right)$

viii $)\left\|F\left(n_{o}+\delta f(p)\right) u\right\|_{\lambda} \leq F\left(n_{o}\right) R+\delta R C\left(R+\|\Psi\|_{1+\lambda}\right)$ 
$i x)\left\|u \cdot \nabla\left(\frac{u}{n_{o}+\delta f(p)}\right)\right\|_{\lambda} \leq C \sum_{i, j} \delta^{i} R^{j}$,

where

$0 \leq i \leq 3$ and $2 \leq j \leq 5$.

Demonstration of Lemma 1:

i)The proof of the first item is the following:

$$
\begin{aligned}
& \text { If }\|p-\Psi\|_{1+\lambda}<R \text { then }|\mathrm{p}(\mathrm{x}, \mathrm{t})|<R+\|\Psi\|_{1+\lambda} \text { now } \\
& \|f(p)\|_{\lambda}=\sup _{Q_{T}}|f(p(x, t))|+\sup _{x, y, t} \frac{|f(p(x, t))-f(p(y, t))|}{|x-y|^{\lambda}} \\
& +\sup _{x, t, s} \frac{|f(p(x, t))-f(p(x, s))|}{|t-s|^{\frac{\lambda}{2}}} \\
& \leq C_{R, \Psi} \sup _{Q_{V}}|p(x, t)|+C_{R, \Psi} \sup _{x, y, t} \frac{|p(x, t)-p(y, t)|}{|x-y|^{\lambda}} \\
& +C_{R, \Psi} \sup _{x, t, s} \frac{|p(x, t)-p(x, s)|}{|t-s|^{\frac{\lambda}{2}}} \\
& \leq C\|p\|_{\lambda} \leq C\left(R+\|\Psi\|_{1+\lambda}\right)
\end{aligned}
$$

ii) The demonstration is very similar to (i).

iii) Making similar counts like in (i) we obtain:

$$
\begin{aligned}
& \left\|F\left(n_{o}+\delta f(p)\right)\right\|_{\lambda}-F\left(n_{o}\right) \leq\left\|F\left(n_{o}+\delta f(p)\right)-F\left(n_{o}\right)\right\|_{\lambda} \\
& \leq \delta C\left(R+\|\Psi\|_{1+\lambda}\right)
\end{aligned}
$$


so $\left\|F\left(n_{o}+\delta f(p)\right)\right\|_{\lambda} \leq F\left(n_{o}\right)+\delta C\left(R+\|\Psi\|_{1+\lambda}\right)$

iv)The demonstration of fourth item is the following:

$$
\begin{aligned}
& n_{o}+\delta f(p) \geq n_{o}-\delta|f(p)| \geq n_{o}-\delta\|f(p)\|_{\lambda} \geq n_{o}-\delta\|p\|_{\lambda} \geq \\
& \geq n_{o}-\delta C\left(R+\|\Psi\|_{1+\lambda}\right) \geq \frac{n_{o}}{2}
\end{aligned}
$$

if, and only if, $\delta \leq \frac{n_{o}}{2 C\left(R+\|\Psi\|_{1+\lambda}\right)}$

v)To demonstrate the fifth item, we only have to develop the $\lambda$-norm of

$$
\frac{1}{n_{0}+\delta f(p)} \text { and use item (i): }
$$

v)To demonstrate the fifth item, we only have to develop the $\lambda$-norm of

$$
\frac{1}{n_{o}+\delta f(p)} \text { and use item (i): }
$$

$$
\| \frac{1}{n_{o}+\delta f(p)}||_{\lambda}=\sup _{Q_{r}}\left|\frac{1}{n_{o}+\delta f(p)}\right|+
$$

$\sup _{x, y, t} \frac{\left|\frac{1}{n_{o}+\delta f(p(x, t))}-\frac{1}{n_{o}+\delta f(p(y, t))}\right|}{|x-y|^{\lambda}}+$

$$
+\sup _{x . t . s} \frac{\left|\frac{1}{n_{0}+\delta f(p(x, t))}-\frac{1}{n_{0}+\delta f(p(x, s))}\right|}{|t-s|^{\frac{\lambda}{2}}}
$$




$$
\begin{aligned}
& \leq C+\delta \sup _{x, y, t, t} \frac{|f(p(x, t))-f(p(y, t))|}{|x-y|^{\lambda}\left|n_{o}+\delta f(p(x, t))\right| n_{o}+\delta f(p(y, t)) \mid}+ \\
& \delta \sup _{x, t, s} \frac{|f(p(x, t))-f(p(x, s))|}{|t-s|^{\frac{\lambda}{2}}\left|n_{o}+\delta f(p(x, t))\right| n_{o}+\delta f(p(x, s)) \mid} \\
& \leq C+\delta C \sup _{x, y, t} \frac{|p(x, t)-p(y, t)|}{|x-y|^{\lambda}}+\delta C \sup _{x, t, s} \frac{|p(x, t)-p(x, s)|}{|t-s|^{\frac{\lambda}{2}}} \leq \\
& \leq C+\delta C\left(R+\|\Psi\|_{1+\lambda}\right)
\end{aligned}
$$

vi) The demonstration of sixth item is analogous to the above:

$$
\begin{aligned}
& \left\|\left(n_{o}+\delta f(p)\right) g\right\|_{\lambda} \leq n_{o}\|g\|_{\lambda}+\delta\|f(p) g\|_{\lambda} \leq n_{o}\|g\|_{\lambda}+\delta C\|p\|_{\lambda}\|g\|_{\lambda} \leq \\
& \leq n_{o}\|g\|_{\lambda}+\delta C\left(R+\|\Psi\|_{1+\lambda}\right)\|g\|_{\lambda}
\end{aligned}
$$

In the second inequality we use the hypothesis about $f$, and in the last we use the hypothesis about $p$.

vii)To obtain the inequality of seventh item, we use (4) and the hypothesis about $f$ and $p$.

viii)To demonstrate the inequality of eighth item, we also use (4) and the hypothesis about $F$ and $p$.

ix)And finally, the ninth item is demonstrated by the following way:

$$
\left\|u \cdot \nabla\left(\frac{u}{n_{0}+\delta f(p)}\right)\right\|_{\lambda} \leq\left\|\frac{u \cdot \nabla u}{n_{0}+\delta f(p)}\right\|_{\lambda}+\left\|u \cdot \nabla\left(\frac{1}{n_{0}+\delta f(p)}\right) u\right\|_{\lambda}
$$


In the right side, the first dot means the product in (2), and the second is the canonical inner product in $I R^{d}$.

The first term of the right side is bounded above in the following way:

$$
\left\|\frac{u \cdot \nabla u}{n_{o}+\delta f(p)}\right\|_{\lambda} \leq\|u\|_{\lambda}\|\nabla u\|_{\lambda}\left\|\frac{1}{n_{o}+\delta f(p)}\right\|_{\lambda}
$$

Now we use items (i) and (ii) above and the hypothesis about $u$ to conclude:

$\leq R^{2} C\left(C+\delta C\|p\|_{\lambda}\right) \leq R^{2} C\left(1+\delta\left(R+\|\Psi\|_{1+\lambda}\right)\right)$

The second one becomes:

$$
\begin{aligned}
& \left\|u \cdot \nabla\left(\frac{1}{n_{o}+\delta f(p)}\right) u\right\|_{\lambda} \leq C\|u\|_{\lambda}^{2}\left\|\nabla\left(\frac{1}{n_{o}+\delta f(p)}\right)\right\|_{\lambda} \\
& =C\|u\|_{\lambda}^{2}\left\|\frac{\delta f^{\prime}(p) \nabla(p)}{n_{o}+\delta f(p)}\right\|_{\lambda} \\
& \leq C \delta\|u\|_{1+\lambda}^{2}\left\|f^{\prime}(p)\right\|_{\lambda}\|\nabla(p)\|_{\lambda}\left\|\frac{1}{n_{o}+\delta f(p)}\right\|_{\lambda}
\end{aligned}
$$

Now using the hypothesis about $u, p$ and $f^{\prime}$; (i) and (ii) we obtain:

$$
\left.\delta R^{2}\left(f^{\prime}(0)\right)+C\left(R+\|\Psi\|_{1+\lambda}\right)\right)\left(R+\|\Psi\|_{1+\lambda}\right)\left(C+\delta C\left(R+\|\Psi\|_{1+\lambda}\right)\right)^{2}
$$

In this way we have proved (ix).

\section{THE EXISTENCE THEOREM}

The general idea of the demonstration is the following:

$$
\text { We will construct sequences }
$$

$p_{m} \in \mathrm{H}^{1+\lambda}\left(Q_{T}\right) ; u_{m} \in\left(\mathrm{H}^{2+\lambda}\left(Q_{T}\right)\right)^{d}$ defined recursively in the way that we can use Theorem 2. And we will obtain uniform limitation of sequences 
$\mathrm{H}^{1+\lambda}\left(Q_{T}\right)$ and $\left(\mathrm{H}^{2+\lambda}\left(Q_{T}\right)\right)^{d}$ respectively. Using compacity results in Hölder's spaces we will obtain convergent subsequences to solution of problem(1).

The sequences are recursively defined in the following way:

$$
\begin{aligned}
& u_{1} \in\left(\mathrm{H}^{1+\lambda}\left(Q_{T}\right)\right)^{d} \text { with condition } u_{1}(x, 0)=0 \forall x \in W \\
& p_{1} \in \mathrm{H}^{1+\lambda}\left(Q_{T}\right) \text { with condition } p_{1}(x, 0)=\Psi(x, 0) \forall x \in \Omega
\end{aligned}
$$

Assuming that $p_{m-1} \in \mathrm{H}^{1+\lambda}\left(Q_{T}\right) ; u_{m-1} \in\left(\mathrm{H}^{1+\lambda}\left(Q_{T}\right)\right)^{d}$ are known we obtain $u_{m}$ and $p_{m}$ satisfying:

$$
\begin{aligned}
& \frac{\partial u_{m}}{\partial t}-\mu \Delta u_{m}+\nabla\left(n_{o}\left(p_{m}-\Psi\right)\right)=\left(n_{o}+\delta f\left(p_{m-1}\right)\right) g_{1} \\
& -\delta f\left(p_{m-1}\right) \nabla\left(p_{m-1}-\Psi\right)- \\
& -\mu F\left(n_{o}+\delta f\left(p_{m-1}\right)\right) u_{m-1}-u_{m-1} \cdot \nabla\left(\frac{u_{m-1}}{n_{o}+\delta f\left(p_{m-1}\right)}\right), \text { in } Q_{T}
\end{aligned}
$$

with:

$$
\left\{\begin{array}{l}
\operatorname{div} u_{m}=0, \text { in } Q_{T} \\
u_{m}(x, 0)=0, \forall x \in \Omega \\
u_{m}(x, t)=0, \forall t \in(0, T) \forall x \in \partial \Omega
\end{array}\right.
$$

This form of definition the sequences is motivated by Solonnikov's Theorem (Theorem 2) which guarantee the existence of sequences.

Observation: Observe that condition (6) of Theorem 2 is satisfied, because if $u_{m-1}$ and $p_{m-1}$ satisfy $p_{m-1}(x, 0)=\Psi(x, 0)$ then $p_{m}$ will satisfy the same thing.

The demonstration is the following: 
The equation linking $p_{m}$ and $p_{m-1}$ is the following:

$$
\begin{aligned}
& \frac{\partial u_{m}}{\partial t}-\mu \Delta u_{m}+\nabla\left(n_{o}\left(p_{m}-\Psi\right)\right)=\left(n_{o}+\delta f\left(p_{m-1}\right)\right) g_{1} \\
& -\delta f\left(p_{m-1}\right) \nabla\left(p_{m-1}-\Psi\right)- \\
& -\mu F\left(n_{o}+\delta f\left(p_{m-1}\right)\right) u_{m-1}-u_{m-1} \cdot \nabla\left(\frac{u_{m-1}}{n_{o}+\delta f\left(p_{m-1}\right)}\right) \text {, in } Q_{T}
\end{aligned}
$$

Taking $t=0$ we obtain:

$$
\begin{aligned}
& \frac{\partial u_{m}(x, 0)}{\partial t}-\mu \Delta u_{m}(x, 0)+\nabla\left(n_{o}\left(p_{m}(x, 0)-\Psi(x, 0)\right)\right)= \\
& -\delta f\left(p_{m-1}(x, 0)\right) \nabla\left(p_{m-1}(x, 0)-\Psi(x, 0)\right)
\end{aligned}
$$

Because $g_{1}(x, 0)=0$ and $u_{m-1}(x, 0)=0$. Now, in agreement with [3] at page 280 , the initial pressure is given by:

$$
\left\{\begin{array}{l}
\Delta\left(n_{o}\left(p_{m}(x, 0)-\Psi(x, 0)\right)\right)=\operatorname{div}\left(-\delta f\left(p_{m-1}(x, 0)\right) \nabla\left(p_{m-1}(x, 0)-\Psi(x, 0)\right)\right) \\
\frac{\partial\left(n_{o}\left(p_{m}(x, 0)-\Psi(x, 0)\right)\right)}{\partial \vec{N}}=\left(-\delta f\left(p_{m-1}(x, 0)\right) \nabla\left(p_{m-1}(x, 0)-\Psi(x, 0)\right)\right) \cdot \vec{N}
\end{array}\right.
$$

Then, if $p_{m-1}(x, 0)=\Psi(x, 0)$ we obtain the system:

$$
\left\{\begin{array}{l}
\Delta\left(n_{o}\left(p_{m}(x, 0)-\Psi(x, 0)\right)\right)=0 ; \forall x \in \Omega \\
\frac{\partial\left(n_{o}\left(p_{m}(x, 0)-\Psi(x, 0)\right)\right)}{\partial \vec{N}}=0 ; \forall x \in \partial \Omega
\end{array}\right.
$$

Then $p_{m}(x, 0)-\Psi(x, 0)=C$, but with the assumptions $p_{m}$ and $\Psi$ have average integral zero in $\Omega$ we obtain that $C=0$.

By this way, we can continue the iteration to obtain $u_{m+1}$, because the condition (6) is satisfied. 
Continuing with the demonstration of Theorem 3 we can, therefore, use estimative (8) in (10) obtained:

$$
\begin{aligned}
& \left\|u_{m}\right\|_{2+\lambda}+\left\|n_{o}\left(p_{m}-\Psi\right)\right\|_{1+\lambda} \leq C\left\|\left(n_{o}+\delta f\left(p_{m-1}\right)\right) g_{1}\right\|_{\lambda} \\
& +\delta C\left\|f\left(p_{m-1}\right) \nabla\left(p_{m-1}-\Psi\right)\right\|_{\lambda} \\
& +\mu C\left\|F\left(n_{o}+\delta f\left(p_{m-1}\right)\right) u_{m-1}\right\|_{\lambda}+C\left\|u_{m-1} \cdot \nabla\left(\frac{u_{m-1}}{n_{o}+\delta f\left(p_{m-1}\right)}\right)\right\|_{\lambda}
\end{aligned}
$$

We suppose now that $\left\|u_{m-1}\right\|_{2+\lambda}<R$ and $\left\|p_{m-1}-\Psi\right\|_{1+\lambda}<R$ and we will estimate, using the Lemma 1, the right side of (11). In this way, we obtain similar uniform limitations for $\left\|u_{m}\right\|_{2+\lambda}$ and $\left\|p_{m-1}-\Psi\right\|_{1+\lambda}$.

Then (11) becomes:

$$
\begin{aligned}
& \left\|u_{m}\right\|_{2+\lambda}+\left\|n_{o}\left(p_{m}-\Psi\right)\right\|_{1+\lambda} \leq C n_{o}\left\|g_{1}\right\|_{\lambda}+\delta C\left(R+\|\Psi\|_{1+\lambda}\right)\left\|g_{1}\right\|_{\lambda}+ \\
& +\delta R C\left(R+\|\Psi\|_{1+\lambda}\right)+R \mu C F\left(n_{o}\right)+\delta R C\left(R+\|\Psi\|_{1+\lambda}\right)+C \sum_{i \cdot j}\left(\delta^{i} R^{j}\right),
\end{aligned}
$$

where $0 \leq i \leq 3$ and $2 \leq j \leq 5$.

Now dividing both sides of inequation above by $n_{0}$, and denoting by $K$ the right side we obtain:

$$
\begin{aligned}
& \frac{\left\|u_{m}\right\|_{2+\lambda}}{n_{o}}+\left\|p_{m}-\Psi\right\|_{1+\lambda} \leq \frac{K}{n_{o}} \\
& \text { If } K / n_{o}<R \text { then }\left\|u_{m}\right\|_{2+\lambda} \leq n_{o} R<R \text { and }\left\|p_{m}-\Psi\right\|_{1+\lambda}<R .
\end{aligned}
$$

Therefore if $\mu C F\left(n_{0}\right)<n_{0}$ we can choose $\left\|g_{1}\right\|_{\lambda}, \delta$ and $R$ small enough for that $K / n_{0}<R$.

In this way we can guarantee that: 


$$
\left\|u_{m}\right\|_{2+\lambda}<R \text { and }\left\|p_{m}-\Psi\right\|_{1+\lambda}<R ; \forall m \in I N
$$

Due to (12) and using Theorem 1, we have

$$
\begin{aligned}
& p_{m_{i}} \rightarrow p \text { in } C^{1,0}\left(Q_{T}\right) \\
& u_{m_{i}} \rightarrow u \operatorname{in} C^{2,1}\left(Q_{T}\right)
\end{aligned}
$$

for subsequences of $p_{m}$ and $u_{m}$; in order to simplify notations we will still denote them by $p_{m}$ and $u_{m}$.

Let pass to the limit the parts of (10):

a) $\frac{\partial u_{m}}{\partial t} \rightarrow \frac{\partial u}{\partial t}$ in $C^{o}\left(Q_{T}\right)$ due to (14).

b) $\Delta u_{m} \rightarrow \Delta u$ in $C^{o}\left(Q_{T}\right)$ due to (14).

c) $\nabla\left(n_{o}\left(p_{m}-\Psi\right)\right) \rightarrow \nabla\left(n_{o}(p-\Psi)\right)$ in $C^{o}\left(Q_{T}\right)$ due to (13).

d) $f\left(p_{m-1}\right) \rightarrow f(p)$ in $C^{o}\left(Q_{T}\right)$ due to (13) and because $f$ is a local Lipschitz function.

e) $F\left(n_{o}+\delta f\left(p_{m-1}\right)\right) \rightarrow F\left(n_{o}+\delta f(p)\right)$ in $C^{o}\left(Q_{T}\right)$ because $f\left(p_{m-1}\right) \rightarrow f(p)$ in $C^{o}\left(Q_{T}\right)$ and $F$ is a local Lipschitz function.

f) $u_{m-1} \cdot \nabla\left(\frac{u_{m-1}}{n_{o}+\delta f\left(p_{m-1}\right)}\right) \rightarrow u \cdot \nabla\left(\frac{u}{n_{o}+\delta f(p)}\right)$ in $C^{o}\left(Q_{T}\right)$ because 


$$
\begin{aligned}
& u_{m-1} \cdot \nabla\left(\frac{u_{m-1}}{n_{o}+\delta f\left(p_{m-1}\right)}\right)_{\lambda}=\frac{u_{m-1} \cdot \nabla u_{m-1}}{n_{o}+\delta f\left(p_{m-1}\right)}+u_{m-1} . \\
& \nabla\left(\frac{1}{n_{o}+\delta f\left(p_{m-1}\right)}\right) u_{m-1}
\end{aligned}
$$

Now, the first part, converges to $\frac{u \cdot \nabla u}{n_{o}+\delta f(p)}$ in $C^{o}\left(Q_{T}\right)$ due to (14) and

(d) above and for the second part, we observe that:

$u_{m-1} \cdot \nabla\left(\frac{1}{n_{o}+\delta f\left(p_{m-1}\right)}\right) u_{m-1}=-u_{m-1} \cdot \frac{\delta f^{\prime}\left(p_{m-1}\right) \nabla\left(p_{m-1}\right)}{\left(n_{o}+\delta f\left(p_{m-1}\right)\right)^{2}} u_{m-1}$

Furthermore $f^{\prime}\left(p_{m-1}\right) \rightarrow f^{\prime}(p)$ in $C^{o}\left(Q_{T}\right)$ due to (13) and because $f^{\prime}$ is a local Lipschitz function. Then we have:

$$
u_{m-1} \cdot \frac{\delta f^{\prime}\left(p_{m-1}\right) \nabla\left(p_{m-1}\right)}{\left(n_{o}+\delta f\left(p_{m-1}\right)\right)^{2}} u_{m-1} \rightarrow u \cdot \frac{\delta f^{\prime}(p) \nabla(p)}{\left(n_{o}+\delta f(p)\right)^{2}} u \text { in } C^{o}\left(Q_{T}\right)
$$

Taking the limit, $m \rightarrow \infty$, in the equation (10) turns to:

$$
\begin{aligned}
& \frac{\partial u}{\partial t}-\mu \Delta u+\nabla\left(n_{o}(p-\Psi)\right)=\left(n_{o}+\delta f(p)\right) g_{1}-\delta f(p) \nabla(p-\Psi) \\
& -\mu F\left(n_{o}+\delta f(p)\right) u- \\
& -u \cdot \nabla\left(\frac{u}{n_{o}+\delta f(p)}\right), \text { in } Q_{T}
\end{aligned}
$$

That is, the equation of problem (1) with the initial and contour condition is satisfied.

Observation: The same results are still valid in $\Omega \times(0, \infty)$, because the constant $C$ in (8) do not depend on $T$, and to take the limit we use an 
argument like Cantor's diagonal approach to conclude that the equation is satisfied in any compact set in $\Omega \times(0, \infty)$.

\section{CONCLUSION}

In this work we have showed conditions and a analytical method to solve a difficult equation which models the flow of an incompresible, homogeneous fluid in a granulated porous media and the general conclusion is if the external force field $g$ is small (in the appropriated Hölder's norm) then we can get a small solution $(u, p)$ in the appropriated norms in Hölder's spaces) for the equation.

\section{REFERENCES}

[1] R.A. Adams, Sobolev Spaces, Academic Press, New York, 1975.

[2] J.G. Heywood, The Navier-Stokes equations: On the existence, regularity and decay of solutions, Indiana University journal, Vol. 29, number 5, 1980, pages 639-681.

[3] J.G. Heywood, R. Rannacher, Finite element approximation of the nonstationary Navier-Stokes problem. I- Regularity of solutions and secondorder error estimates for spatial discretization, SIAM, J. Numer. anal., Vol.19, N.2, pages 275-211, 1982.

[4] J. Prieus Du Plessis and J.H. Masliyah, Flow through isotropic granular porous media, Transport in porous media, number 6, pages 207-221, 1991.

[5] V.A. Solonnikov, Estimates for solutions of nonstationary Navier-Stokes equations, Journal of Soviet Mathematics, Vol. 8, number 4, pages 467-529, 1977. 
[6] R. Temam, Navier-Stokes equations-Theory and numerical analysis (revised edition), North-Holland Publ. comp., Amsterdam, 1979.

[7] W. von Wahl, The Equations of Navier-Stokes and Abstract Parabolic Equations, Fried. Vieweg \&\& Sons, Braunschweig/Wiesbaden, 1985. 